Research Article

\title{
Do otoliths modulate caloric response? what do vemps and cdp measure? what do these tests tell us?
}

\begin{abstract}
Objective: it has been documented in the literature that caloric responses with the lateral semicircular canal in a horizontal position are reduced, but not absent. This paper addresses what contributions to the caloric response, if any are made by the otoliths, and what influences on caloric response might be seen in patients with otolithic pathology.

Study design: a prospective case series study.

Setting: Tertiary and quaternary care teaching hospital.

Patients: two normal subjects underwent "horizontal" calorics to document caloric response with the lateral semicircular canal in the horizontal position. This assessment was also carried out on eight sequential patients who were documented to have otolithic disease (as evidenced by abnormal VEMP assessment).

Interventions: Two sets of caloric tests (standard and horizontal) were carried out on our subjects. Patients underwent identical caloric assessment after CVEMP and OVEMP assessment.

Main outcome measures: VEMPs were scored as either normal or abnormal for each parameter we assess. Calorics were scored using the standard slow phase velocity measurements for each one.

Results: Patients with otolithic abnormalities showed different caloric results in the horizontal position than is suggested in the literature and was shown by our two normal subjects.

Conclusion: Caloric responses are modulated and in patients with otolithic pathology, semicircular canal function is modulated, as caloric responses are different in patients than in normals when the superior semicircular canal orientation to gravity is changed. Our results suggest that the otoliths do in fact mediate caloric responses.
\end{abstract}

Keywords: cvemps, ovemps, horizontal bithermal baloric, phillipszoon-jongkees formula, otometrics
Volume 3 Issue 2 - 2015

\author{
Longridge NS, ${ }^{1,2}$ Mallinson Al, ${ }^{1,2}$ Pothier DD ${ }^{3}$ \\ 'Neuro-otology unit, Vancouver General Hospital, Canada \\ ${ }^{2}$ Division of Otolaryngology, Department of Surgery, University \\ of British Columbia, Canada \\ ${ }^{3}$ Department of Otolaryngology- head and neck surgery, \\ University of Toronto, Canada
}

Correspondence: NS Longridge, Neuro-otology unit, Vancouver General Hospital, 4th floor - 2775 Laurel Street Vancouver, BC V5Z IM9, Canada, Tel 604-875-4I II Email nslomgridge@hotmail.com

Received:September 02, 2015 | Published: September 16, 2015

\section{Introduction}

Vestibular evoked myogenic potentials (CVEMPs and OVEMPs) are newer clinically accepted measures of the macular systems of the inner ear. When patients have abnormal VEMP testing this means that there is something wrong with their macula system. The specific symptoms associated with early and late N1 and P1 latencies, altered amplitudes and Interaural amplitude differences are not yet established. It is a reasonable assumption that, as these tests assess the macula, an abnormality in any of these parameters indicates otolithic dysfunction. Standard caloric testing uses warm and cold water, and the slow phase of nystagmus is measured with the lateral semicircular canal in a vertical position. Warm water induces nystagmus with the fast phase towards the irrigated side; the opposite is true for cold water. The mnemonic "COWS"; - "cold opposite; warm same" - is now memorized by medical students and residents, and the nystagmus velocity is analyzed using the well known Phillipszoon-Jongkees formula. ${ }^{1}$ A caloric study by Coats and $\mathrm{Smith}^{2}$ was carried out with the lateral semicircular canal in a vertical and a horizontal position, and showed that there was usually a slight, but definite COWS response in this horizontal position. They suggested that the otoliths were playing a role in this unexpected response; while they knew that otoliths do not generate nystagmus, they suggested that otolithic input may modify nystagmus generated by the semicircular canals. Scherer \& Clark $^{3}$ repeated this study using the stimulus used in NASA space experiments, which was a hot air irrigation of 44 degrees Celsius on one side and simultaneous contra lateral cold air at 15 degrees Celsius. They tested a total of 26years in this manner.

Table I(A) Horizontal Warm Calorics (Coats and Smith) (2) h/v ratio of patients one to four $=2.0$

\begin{tabular}{llll}
\hline Subject & Vertical & Horizontal & Ratio \\
\hline $\mathrm{I}$ & 25 & 10 & 2.5 \\
2 & 21 & 5 & 4.2 \\
3 & 20 & 12 & 1.7 \\
4 & 20 & 15 & 1.3 \\
Total & 86 & 42 & \\
Average & 21.5 & 10.5 & 2 \\
Outlier & 33 & -7 &
\end{tabular}

Table I(B) Horizontal Cold Calorics (Coats and Smith) (2) h/v ratio of patients one to four $=2.4$

\begin{tabular}{llll}
\hline Subject & Vertical & Horizontal & Ratio \\
\hline & 16 & 5 & 3.2 \\
2 & 14 & 6 & 2.3 \\
3 & 10 & 5 & 2 \\
4 & 10 & 5 & 2 \\
Total & 50 & 21 & \\
Average & 12.5 & 5.3 & 2.4 \\
Outlier & 31 & -8 & \\
\hline
\end{tabular}


The average response (slow phase velocity for the lateral semicircular canal in a vertical position) was 32 degrees per second and for the lateral semicircular canal in a horizontal position was 10 degrees per second. In summary, the ratio of the "maximum response" (in the vertical position) to the "minimum response" (canal in the horizontal position) was 3.2. (Table 1). We carried out two different studies. In our first study, two of the authors of this paper undertook the "horizontal bithermal caloric test" using the conventionally accepted stimulus of $250 \mathrm{ml}$. of water at 44 degrees and 30degrees infused over a 30 second period ${ }^{4}$ (performed with patient sitting $30^{\circ}$ head down) (Table 2). This precluded the necessity of trying to talk normal subjects into the unpleasant bithermal test. In our second preliminary study, patients with an abnormal macula system (as shown by abnormal VEMPS) were assessed with the head vertical and horizontal caloric test and compared results to normal subjects in the literature (Table 2).

\section{Methods}

\section{CVEMP technique}

Our CVEMP assessment technique involves having the patient sitting comfortably and leaning forward, resting the chin on an inflated blood pressure cuff, which is positioned on an adjustable height hospital food trolley. Software in our machine (GN Otometrics, Schaumburg IL) includes an EMG patient feedback system and allows for the setting of an EMG flexion window so that responses are only recorded when the SCM is flexed between 30 and $70 \mu \mathrm{V}$ (the window recommended by the manufacturer, and a flexion level that is easy to maintain consistently). This eliminates any differences created by "side to side" flexion as our patients are in an identical position and carry out an identical task regardless of which side is being measured. We find that patient instruction is easy as the patient task is identical regardless of which side is being measured, and EMG monitoring is carried out with ease. We use a sound stimulus with insert earphones, which results in a high Interaural attenuation, meaning that the side stimulated is the side from which the response arises. Insert earphones have a wider interaural attenuation than other earphone types and the attenuation at $500 \mathrm{~Hz}$ is at least $90 \mathrm{~dB} \mathrm{HL}^{5}$ (i.e., making a unilaterally supplied sound stimulus very specific). We present $500 \mathrm{~Hz}$ tone bursts at $5.1 \mathrm{~Hz}$, and 125 runs are completed. Two trials are completed, and averaged. Patients with an air bone gap at $500 \mathrm{~Hz}>10 \mathrm{~dB}$ are excluded from clinical studies.

\section{OVEMP technique}

In the same way we have tried to standardize CVEMPs, we have made our OVEMP technique as reproducible as possible. It has been shown by $\mathrm{O}^{\prime} \mathrm{Neil}^{6}$ that having participants sit upright during OVEMP testing is optimum for achieving the best threshold possible. OVEMP measurement is carried out by instructing the patient to elevate gaze to a level of 30 degrees, by fixating a stimulus in the upper wall of the room. International norms for VEMP results have been published. As recommended, ${ }^{7}$ we nor med our equipment with 40 normal people who had no history of dizziness or imbalance, and found that results were extremely close to those of other international assessors for this particular stimulus, ${ }^{8}$ (Tables $3 \& 4$ ). Using the same strategy for abnormal as employed using the gold standard measurement criteria (the Philipszoon Jongkees formula ${ }^{1}$ of 2 standard deviations from the normal; "abnormal VEMPS" are outside of this range. Pathology is indicated by abnormalities in P1 and N1 latency and by amplitudes above and below 2 standard deviations from the normal. We also use the same criteria (i.e. 2 S.D) for interaural amplitude difference.

\section{Vertical and horizontal calorics" technique}

Eight patients with documented VEMP abnormalities underwent standard caloric assessment with the lateral semicircular canal vertical (Table 2). Calorics were then repeated with head forward $30^{\circ}$ from vertical; this renders the lateral semicircular canal horizontal. The caloric total in both conditions were compared.

Caloric response and VEMP in two different positions (Standard and $90^{\circ}$ offset) and VEMP abnormalities. U: Unilateral finding; B: Bilateral finding; A: Amplitude; L: Latency; IAR: Interaural Amplitude Ratio; ABS: Bilaterally Absent.

\section{Results}

Our preliminary study included 8 patients with known vestibular disease related to trauma; they had all suffered some kind of trauma that we define as "vestibular trauma"a sudden decelerative event (usually a whiplash type of injury) (Table 2). Two patients had abnormal calorics. Posturography was carried out in all patients, but was not helpful in assessment, as it was normal in 4 patients and showed a nonspecific abnormality in the other four. VEMP assessments were carried out in all patients and all had abnormalities. In 6 of the patients a bilateral abnormality was documented. CVEMPs-One patient had normal CVEMPs. One patient had bilaterally absent CVEMPs, one had unilaterally absent CVEMPs, two had bilateral latency abnormalities, and three had an amplitude asymmetry (using the "Phillipszoon-Jongkees" technique for assessing VEMP response). OVEMPs-One patient had normal OVEMPs. Two patients had bilaterally absent OVEMPs, three had latency abnormalities and two had an amplitude asymmetry. Horizontal calorics were present in all of our patients and were inappropriately robust in nature, as opposed to the relatively low responses seen in our two normals and in the normals studied by others. ${ }^{2,3}$

\section{Discussion}

The bithermal caloric test is a notoriously unpleasant test and finding normal volunteers to undertake is a challenge. Table 5 shows that there was definite but minimal response to horizontal calorics in our two volunteers. This is markedly similar to the results of reported by Scherer \& Clark, ${ }^{3}$ which were duplicated by Coats \& Smith $^{2}$ in Table one. Interestingly, one of Coats and Smith's subjects (subject 5) was an outlier; it is possible that this patient had otolithic pathology to account for this (but at that time no assessments existed to measure otolithic function). At that time, the subtle complaints now recognized as being from otolithic disease were unrecognized. Although the vertical/horizontal ratios reported in the literature were about 2.4:1, our two normals had ratios of at least 9:1. This may come from the different calculation techniques used by Coats and Smith. In calculating response, they identified a "maximum caloric nystagmus" period which always consisted of at least 5 beats and usually about 20 beats, ${ }^{2}$ and calculated the average slow phase velocity of this period. This would tend to reduce the maximum response reported (when compared to the standard " 3 beats of maximum velocity in 10 seconds" technique outlined by Barber and Stockwell. ${ }^{4}$ As this paper is about caloric function, it is not the place to discuss the complexities of VEMPS in detail. Over the years, caloric response has been explained using a simple lever process. We hypothesize that this explanation is not appropriate and ignores the biological nature of the very complex vestibular system. The response with the lateral semicircular canal vertical is present but over a 30degree range to either side these responses change very little. ${ }^{2}$ Whatever causes this response must be a broad effect on the cupula stimulus, perhaps indicating that there is 
some flexible stretching of the crista, and directional translation of this response, as alluded to by Coats \& Smith. ${ }^{2}$

Coats and Smith reported an "outlier"; possibly a subject with vestibular otolithic disease as one of their normal people. Marked disruption of performance resulting from otolithic disturbance has also been reported by Black et al., ${ }^{9}$ whose study group included one subject performing far below mean performance, and this related to another assessment stimulating his otoliths that he had been subjected to a few days before. The suggestion was that stimuli to the otoliths resulted in a decreased ability of them to integrate the rotational signals in an appropriate manner, and this disrupted postural control. Although this is a different experimental paradigm, to us it reflects the role of the otoliths as integrators of any vestibular signal. The biological process of the crista placed transversely across the ampulla provides it with variable ability to stretch and be stimulated by cupula movement not in a single vector plane, but in 3 vectors in space. The original work by Jongkees in ${ }^{10}$ has been discussed and expanded upon by several investigators. ${ }^{11-13}$ Fernandez \& Goldberg ${ }^{14}$ showed in 1976 that head translation as sensed by the otoliths is also an important stimulus for compensatory eye movement. Our study supports their work and also the work of Coats \& $\mathrm{Smith}^{2}$ who suggested that, during clinical assessment, otolithic dysfunction could modify the caloric response in the horizontal plane, despite the fact that standard caloric tests indicate normal lateral semicircular canal function. Based on the studies by Scherer and Clarke and on our studies in normal people (and also based on the physiology of the vestibular system as we understand it), it seems that, although the horizontal caloric response is present, it is of low velocity. Our study indicates that patients with VEMP abnormalities have abnormal caloric responses at $90^{\circ}$ to lateral semicircular canal vertical which are out of proportion to the normal small "COWS response" with the lateral semicircular canal horizontal.

Table 2 Horizontal and Vertical Calorics (otolithic patients)

\begin{tabular}{lllllllll}
\hline Subject & \multicolumn{2}{c}{ Caloric Total (vert/horiz) } & V/h ratio & Trauma & Cvemp & Ovemp & Cal. & Equi \\
\hline \multicolumn{1}{l}{ Initials age/sex } & & & & & & \\
2 & BD 44M & 2425 & 0.96 & WADx2 & latency & IAR & $\mathrm{N}$ & $\mathrm{N}$ \\
3 & DS 40F & 4965 & 0.75 & WAD & ABS & ABS & $\mathrm{N}$ & NonsP \\
4 & FC 5IM & 57 I8 & 3.2 & HI+WAD & IAR & latency & $\mathrm{N}$ & $\mathrm{N}$ \\
5 & JR 60M & 249 & 2.7 & HI & latency & ABS & $\mathrm{N}$ & nonsp \\
6 & SR 47M & 6014 & 4.3 & WAD & IAR & NORMAL & $\mathrm{N}$ & $\mathrm{N}$ \\
7 & OS 58F & 356 & 4.2 & HI+WAD & IAR & latency & $\mathrm{N}$ & $\mathrm{N}$ \\
8 & BK 62 M & 4025 & 1.6 & WAD & UA & BL & ABN & 234 \\
& DM 43M & 3343 & 0.76 & WADx2 & NORMAL & IAR + L & ABN & $6-M a r$
\end{tabular}

Table 3 Cervical Vestibular Evoked Myogenic Potential (CVEMPs) Normative data comparison

\begin{tabular}{llll}
\hline Subject & Mallinson \& Longridge [16] & Vanspauwen \&Wuyts [18] & Nguyen et al. [8] \\
\hline PI Latency & $15.4( \pm .1 .5) \mathrm{msec}$ & $15.4( \pm .1 .5) \mathrm{msec}$ & \\
& & $24.1( \pm 2.0) \mathrm{msec}$ & $\begin{array}{l}13.5( \pm .1 .4) \mathrm{msec} \\
21.9( \pm .3 .2) \mathrm{msec} \\
\text { NI Latency }\end{array}$ \\
Amplitude (normal) & $24.1( \pm 2.0) \mathrm{msec}$ & $54-505 \mu \mathrm{V}$ & not reported \\
& $54-505 \mu \mathrm{V}$ & $<34.5 \%$ & $<31.6 \%$ \\
Interaural Amplitude ratio (IAR) & $<34.5 \%$ & $<$
\end{tabular}

Table 4 Ocular Vestibular Evoked Myogenic Potential (OVEMPs) Normative data comparison

\begin{tabular}{llll}
\hline Subject & Mallinson \& Longridge [16] & Nguyen et al. [8] & Xie et al. [19] \\
\hline NI Latency & $10.35( \pm .66) \mathrm{msec}$ & $10.35( \pm .5 \mathrm{I}) \mathrm{msec}$ & \\
& & & $10.35( \pm .66) \mathrm{msec}$ \\
PI Latency & $15.18( \pm 1.07) \mathrm{msec}$ & $15.48( \pm .82) \mathrm{msec}$ & $15.18( \pm 1.07) \mathrm{msec}$ \\
Amplitude (normal) & $<14.84 \mu \mathrm{V}$ & Not Reported & $<14.84 \mu \mathrm{V}$ \\
& $<31 \%$ & $<31 \%$ & $<31 \%$ \\
Interaural Amplitude ratio (IAR) & & &
\end{tabular}

Table 5 Caloric Results in Our Two Normal Subjects

\begin{tabular}{llll}
\hline & Vertical hot & Horizontal hot & Ratio \\
\hline $69 \mathrm{M}$ & 73 & 7 & 10.3 \\
$62 \mathrm{M}$ & 62 & 6 & 10.4 \\
\hline
\end{tabular}

All of our eight study patients had abnormal VEMPs. Most patients had complaints which were characterized in the literature as being otolithic in origin (i.e. vague imbalance, "walking on foam", "feeling intoxicated", or newly developed visual vestibular mismatch). ${ }^{15,16}$ This symptom set has recently been classified as "visually induced dizziness". ${ }^{17-19}$ We found that in patients with abnormal VEMPs and normal caloric response, the horizontal calorics resulted in caloric responses greatly different to what would have been predicted in normals. Table 2 lists the VEMP abnormalities. Posturography as a diagnostic entity was not helpful, as it was invariably normal. This was not surprising to us, as a disruption of otolithic/semicircular canal interaction (the purpose of which is to generate a VOR response in the form of an appropriate nystagmus) would not be picked up by an assessment designed to assess vestibulospinal reflexes. (Interestingly, posturography was abnormal in two of the three patients with caloric reductions; this is to be expected). Table 2 also lists totaled caloric responses at $30^{\circ}$ head up and $60^{\circ}$ head down horizontal. The discrepancy in these results when compared to normal subjects and the inappropriately robust nature of the horizontal response confirms the probable likelihood of otolithic modulation of caloric responses. Seven of our 8 patients had bilateral abnormalities.

This small "horizontal caloric study" results in more questions than answers. The future potential for using caloric testing at other 
head positions than $30^{\circ}$ head up needs to be determined by further evaluation of angle of lateral semicircular canal during caloric assessment related to gravity and comparison to the various types of VEMP abnormalities to see which symptoms are associated with particular VEMP abnormalities and how caloric responses are altered. If abnormalities on VEMP testing are associated with measured caloric responses which are not in keeping with the minimal response sown in normals, this may throw light on how the caloric test is modulated by the otoliths, and perhaps what otolith pathology is indicated by the various VEMP abnormalities which are associated with this modulation. It must be kept in mind (and this paper recognizes) that caloric testing is completely physiological and therefore caution with respect to interpretation of the significance of this finding in this study is necessary. Despite this, it has stood the test of time and of clinical relevance. This paper shows that if the VEMPS are abnormal, then the caloric response with the head at different positions to the optimal $30^{\circ}$ head up position results in responses which do seem to be attenuated as one might expect and has been shown in normal people. This indicates that there is modulation of this response by the otoliths. If one extrapolates this finding to physiological vestibule-ocular reflex function, the conclusion we draw is that the otoliths serve to modify semicircular canal activity.

\section{Acknowledgments}

None.

\section{Conflicts of interest}

Author declares there no conflicts of interest.

\section{Funding}

None.

\section{References}

1. Jongkees, LBW, Philipszoon AJ. Electronystagmography. Acta OtoLaryngol. 1964;189(Suppl):7-111.

2. Coats AC and Smith SY . Body position and the intensity of caloric nystagmus. Acta oto-laryngologica. 1967;63(2-3):515-532.

3. Scherer H, Clarke AH. Thermal stimulation of the vestibular labyrinth during orbital flight. Arch Otorhinolaryngol. 1987;244(3):159-166.

4. Barber HO, Stockwell CW. Manual of Electronystagmography $\left(2^{\text {nd }}\right.$ edn $)$ CV Mosby, St. Louis, Libraries Australia. 1980.

5. Katz J, Medwetsky L, Burkard R, et al.Handbook of clinical audiology. $\left(6^{\text {th }}\right.$ edn), Lippincott Williams and Wilkins Section 1 Introduction and basic tests and procedures. Baltimore, USA. 2009. p.189-221.
6. O'Neil AR. Ocular vestibular evoked myogenic potentials (oVEMP) using air conducted sound: Effect of body position on threshold". Independent Studies and Capstones. Program in Audiology and Communication Sciences, Washington University School of Medicine. 2010. p. 601.

7. Janky KL, Nguyen KD, Welgampola M, et al.Air-conducted oVEMPs provide the best separation between intact and superior canal dehiscent labyrinths. Otol Neurotol. 2013;34(1):127-134.

8. Nguyen KD, Welgampola MS, Carey JP. Test-retest reliability and age-related characteristics of the ocular and cervical vestibular evoked myogenic potential tests. Otol Neurotol. 2010;31(5):793-802.

9. Black FO, Paloski WH, Reschke MF, et al. Disruption of postural readaptation by inertial stimuli following space flight. $J$ Vest Res. 1999;9(5):369-378.

10. Jongkees LBW. Origin of the caloric reaction of the labyrinth. Arch Otolaryngol. 1948;48(6):645-657.

11. Stahle J. Controversies on the caloric response. From Barany's theory to studies in microgravity. Acta Otolaryngol . 1990;109(3-4):162-167.

12. Pau HW, Limberg W. Fluid kinetics of endolymph during rotation. Acta Otolaryngol. 1990;109(1-2):7-10.

13. Wit HP, Spoelstra HA, Segenhout JM. Barany's theory is right, but incomplete. Acta Otolaryngol. 1990;109(1-2):1-6.

14. Fernandez C, Goldberg JM. Physiology of peripheral neurons innervating otolith organs of the squirrel monkey. I. Response to static tilts and to long-duration centrifugal force. J Neurophysiol. 1976;39(5):970-984.

15. Lee JD, Park MK, Lee BD, et al. Otolith unction in patients with head trauma. Eur Arch Otorhinolaryngol. 2011;268(10):1427-1430.

16. Mallinson AI, Longridge NS. Visual vestibular mismatch in work related vestibular injury. Otol Neurotol. 2005;26(4):691-694.

17. Bisdorff A, Von Brevern M, Lempert T, et al. Classification of vestibular symptoms: Towards an international classification of vestibular disorders. J Vest Res. 2009;19(1-2):1-13.

18. Vanspauwen R, Wuyts FL, Van de Heyning PH. Vestibular evoked myogenic potentials: Test-retest reliability and normative values obtained with a feedback method for the sternocleidomastoid muscle contraction. $J$ Vest Res. 2009;19(3-4):1-9.

19. Xie SJ, Xu Y, Bi HZ, et al. Ocular vestibular-evoked myogenic potentials in healthy pilots and student pilots. Aviat Space Environ Med. 2011;82(7):729-733. 